\section{Brain Diffusion-Weighted and Diffusion Tensor Imaging Findings in an Infant with Biotinidase Deficiency}

A previously healthy 7-week-old male infant was admitted because of clonic movements of the upper limbs. Findings of a neurologic examination were normal. His psychomotor development was appropriate for his age. Brain MR imaging showed supra- and infratentorial white matter signal-intensity abnormality, suggestive of edema, with frontal predominance and peripheral U-fiber involvement. Myelination was slightly diminished in the posterior limbs of the internal capsules, also compatible with edema. The corpus callosum was spared. Deep gray matter structures had a normal appearance. No malformations were detected. Diffusion-weighted imaging (DWI, $b=1000 \mathrm{~s} / \mathrm{mm}^{2}$ ) and diffusion tensor imaging revealed symmetric moderately reduced diffusion with abnormally increased fractional anisotropy in the centra semiovalia and perirolandic white matter (Fig 1). MR spectroscopy (regions of interest positioned at the left centrum semiovale and basal nuclei; TE $=30$ and 135 ms; not shown) showed a lactate doublet at $1.33 \mathrm{ppm}$.

Laboratory investigation demonstrated elevated plasma lactate (19.6 $\mathrm{mg} / \mathrm{dL}$; reference range, 3.1-12.0 $\mathrm{mg} / \mathrm{dL}$ ) and urinary 3-hydroxyisovaleric acid ( $1786 \mu \mathrm{mol} / \mathrm{mmol}$ of creatine; reference range, 10-67 $\mu \mathrm{mol} / \mathrm{mmol}$ of creatine) levels and absent plasma biotinidase activity. Biotin supplementation (5 mg per day, by mouth) was started. No further seizures were noted. A follow-up brain MR imaging at 3 months of age showed normalization of the white matter signal intensity. The child was neurologically and developmentally normal at 10 months of age.

Biotinidase converts biotin, an essential water-soluble vitamin, into its bioavailable form. Biotinidase deficiency is a rare autosomal recessive disorder, with an estimated prevalence of approximately 1:60,000 neonates, ${ }^{1}$ causing multiple carboxylase deficiencies (for which biotin is a coenzyme) and leading to energy depletion, due to impaired catabolism of several branched chain amino acids, gluconeogenesis, and fatty acid synthesis. ${ }^{2}$ Neurologic involvement in biotinidase deficiency manifests in the first few years of life, with seizures, optic atrophy, and sensorineural hearing loss. ${ }^{3}$ Conventional MR imaging and MR spectroscopy features reflect the known pathophysiologic mechanisms in this deficiency. Prolonged $\mathrm{T} 1$ and $\mathrm{T} 2$ of the white matter may be explained by diffuse interstitial edema. The lactate peak on MR spectroscopy is related to the conversion to anaerobic metabolism. ${ }^{4,5}$

A previous description of a patient with biotinidase deficiency ${ }^{5}$ demonstrated markedly reduced diffusion in the brain stem, middle cerebellar peduncles, splenium of the corpus callosum, posterior limbs of the internal capsules, corona radiata, and parieto-occipital gray matter. In our patient, a different pattern was observed, with moderately reduced diffusion and increased fractional anisotropy in the perirolandic white matter and centra semiovalia. This could be due to accelerated myelination of those areas, induced by the repeated neuronal electric activity of the seizures, as was previously reported in children with Stürge-Weber syndrome $^{6}$ and in experimental studies in mice. ${ }^{7}$ Vacuolating myelinopathy and myelin edema were previously described in neuropathologic studies in biotinidase deficiency. ${ }^{3}$ However, in our patient, these seem an unlikely explanation for the imaging findings because intramyelinic edema usually causes markedly reduced water diffusion and decreased fractional anisotropy in white matter. ${ }^{8}$

\section{References}

1. Wolf B. Worldwide survey of neonatal screening of biotinidase deficiency. J Inherit Metab Dis 1991;14:923-37

2. Wolf B. Disorders of biotin metabolism. In: Scriver CR, Beaudet AL, Valle D, et al, eds. The Metabolic and Molecular Bases of Inherited Disease. 8th ed. New York: McGraw-Hill; 2001:3935-62

3. van der Knaap MS, Valk J. Multiple carboxylase deficiency. In: Magnetic Resonance of Myelination and Myelin Disorders. 3rd ed. Berlin: Springer-Verlag; 2005:248-51

4. Schürmann M, Engelbrecht V, Lohmeier K, et al. Cerebral metabolic changes in biotinidase deficiency. J Inherit Metab Dis 1997;20:755-60

5. Desai S, Ganesan K, Hegde A. Biotinidase deficiency: a reversible metabolic encephalopathy — neuroimaging and MR spectroscopic findings in a series of four patients. Pediatr Radiol 2008;38:848-56

6. Jacoby CG, Yuh WT, Afifi AK, et al. Accelerated myelination in early StürgeWeber syndrome demonstrated by MR imaging. J Comput Assist Tomogr 1987;11:226-31

7. Demerens C, Stankoff B, Logar M, et al. Induction of myelination in the centra nervous system by electrical activity. Proc Natl Acad Sci U S A 1996;93:9887-92

8. Patay Z. Diffusion-weighted MR imaging in leukodystrophies. Eur Radiol 2005; $15: 2284-303$

J.P. Soares-Fernandes Z. Magalhães

J.F. Rocha

Department of Neuroradiology Hospital de S. Marcos

Braga, Portugal

A.J. Barkovich

Department of Radiology and Biomedical Imaging University of California San Francisco, Calif

DOI 10.3174/ajnr.A1703
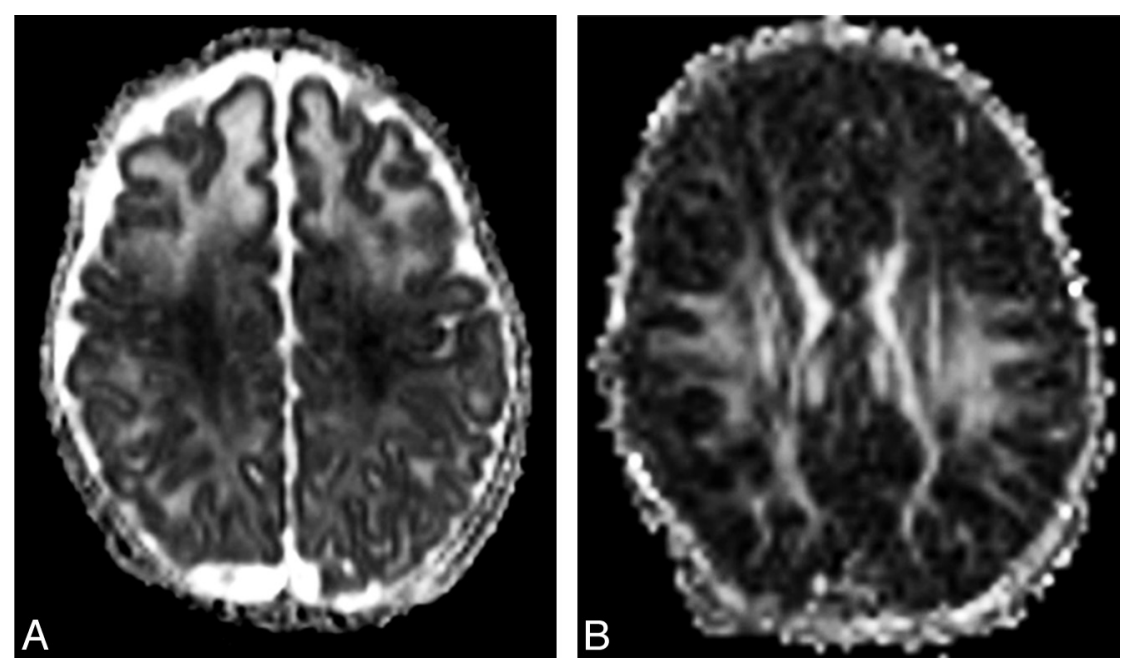

Fig 1. $A$, Apparent diffusion coefficient (ADC) map demonstrates decreased signal intensity in the perirolandic white matter and centrum semiovalia. ADC value, measured in the left centra semiovale at the MR imaging workstation (Syngo Siemens, Erlangen, Germany) is reduced $\left(0.87 \times 10^{-3} \mathrm{~mm}^{2} /\right.$ $\mathrm{s})$; in an age-matched control (not shown), $\mathrm{ADC}$ measured in the same location was $1.4 \times 10^{-3} \mathrm{~mm}^{2} / \mathrm{s}$. Note also increased water diffusion in the remaining hemispheric white matter, with frontal predominance, suggestive of interstitial edema. $B$, Fractional anisotropy map shows increased anisotropy in the perirolandic white matter and centra semiovalia. 